\title{
Gene Expression Profiling of Bronchoalveolar Lavage Cells during Aspergillus Colonization of the Lung Allograft
}

\author{
S. Samuel Weigt, MD ${ }^{1}$, Xiaoyan Wang, PhD ${ }^{1}$, Vyacheslav Palchevskiy, $\mathrm{PhD}^{1}$, Naman Patel, \\ BS $^{1}$, Ariss Derhovanessian, MD¹, Michael Y. Shino, MD ${ }^{1}$, David M. Sayah, MD, PhD ${ }^{1}$, \\ Joseph P Lynch III, MD1, Rajan Saggar, MD ${ }^{1}$, David J Ross, MD ${ }^{1}$, Bernie M. Kubak, MD, \\ PhD $^{1}$, Abbas Ardehali, MD ${ }^{2}$, Scott Palmer, $\mathbf{M D}^{3}$, Shahid Husain, $\mathbf{M D}^{4}$, and John A. Belperio, \\ $M D^{1}$ \\ ${ }^{1}$ Department of Medicine, David Geffen School of Medicine, University of California, Los Angeles, \\ California \\ 2Department of Surgery, David Geffen School of Medicine, University of California, Los Angeles, \\ California \\ ${ }^{3}$ Department of Medicine, Duke University, Durham, North Carolina, USA \\ ${ }^{4}$ Department of Medicine, Division of Infectious Diseases, University of Toronto, Toronto, Ontario, \\ Canada
}

\section{Abstract}

Background-Aspergillus colonization after lung transplant is associated with an increased risk of chronic lung allograft dysfunction (CLAD). We hypothesized that gene expression during Aspergillus colonization could provide clues to CLAD pathogenesis.

\begin{abstract}
Methods-We examined transcriptional profiles in 3 or 6-month surveillance bronchoalveolar lavage fluid cell pellets from recipients with A. fumigatus colonization $(\mathrm{n}=12)$ and without colonization ( $\mathrm{n}=10)$. Among the Aspergillus colonized, we also explored profiles in those who developed CLAD $(n=6)$ or remained CLAD free $(n=6)$. Transcription profiles were assayed with the HG-U133 Plus 2.0 microarray (Affymetrix). Differential gene expression was based upon an absolute fold difference $\geq 2.0$, and unadjusted P-value $<0.05$. We used NIH DAVID for functional analyses, with false discovery rates $<5 \%$ considered significant.
\end{abstract}

Results-Aspergillus colonization was associated with differential expression of 489 probe sets, representing 404 unique genes. "Defense response" genes and genes in the "cytokine-cytokine receptor" KEGG pathway were notably enriched in this list. Among Aspergillus colonized patients, CLAD development was associated with differential expression of 69 probe sets, representing 64 unique genes. This list was enriched for genes involved in "immune response" and

Corresponding Author: S. Sam Weigt, UCLA Med-Pul \& Critical Care, BOX 951690, 37-131 CHS, Los Angeles, CA 90095-1690, sweigt@mednet.ucla.edu.

Authorship: All authors contributed to the interpretation of data, were involved in revising important intellectual content, and approved the final version for publication. In addition, SSW, XW, VP, and JAB contributed to the design of the work, data acquisition and analysis, and drafting of important intellectual content. SSW, AD, MYS, DMS, JPL, RS, DJR and JAB additionally provided bronchoalveolar lavage samples from which gene expression data were obtained.

Disclosures: The authors declare no conflicts of interest. 
"response to wounding", among others. Notably, both chitinase 3-like-1 and chitotriosidase were associated with progression to CLAD.

Conclusion-Aspergillus colonization is associated with gene expression profiles related to defense responses including cytokine signaling. Epithelial wounding, as well as the innate immune response to chitin that is present in the fungal cell wall, may be key in the link between Aspergillus colonization and CLAD.

\section{Introduction}

Lung transplant is a therapeutic option for end-stage pulmonary disorders, but long-term survival is dependent upon remaining free from chronic lung allograft dysfunction (CLAD), which affects greater than $50 \%$ of recipients within 5 years. ${ }^{1}$ The predominant CLAD phenotype manifests as progressive airflow obstruction and is termed bronchiolitis obliterans syndrome (BOS). ${ }^{2,3}$ While acute rejection (AR) is the best described risk factor, there is growing evidence that alloimmune independent insults such as ischemia reperfusion, gastroesophageal reflux disease, and respiratory infections promote the development or progression of CLAD. ${ }^{4-12}$ Although the mechanism as to how these insults lead to CLAD is incompletely understood, a commonly held hypothesis is that injury to the pulmonary epithelium induces wound repair mechanisms that become dysregulated in the alloimmune environment, ultimately leading to chronic fibroproliferation and progressive graft dysfunction. ${ }^{13}$

Aspergillus is a ubiquitous mold that produces conidia (spores) that can be inspired during respiration. Once deposited in a suitable environment such as the lung allograft, the conidia may germinate and proliferate in the mycelial form capable of causing invasive infection. While the normal host usually clears infection at a very early stage, immune suppression, impaired mucociliary clearance, relative tissue ischemia, and increased tissue iron from recurrent micro-hemorrhage make the lung allograft especially vulnerable to Aspergillus infections. ${ }^{14-16}$ While invasive disease is more common after lung transplant than with other solid organ transplants, it still affects only a small percentage of lung recipients. ${ }^{17,18}$ In contrast, asymptomatic Aspergillus infection or "colonization" occurs in approximately onethird of lung transplant recipients within 1 year. ${ }^{18}$ Importantly, we have previously shown that Aspergillus colonization is associated with an increased risk of chronic lung allograft dysfunction (CLAD). ${ }^{18}$ Furthermore, the risk of CLAD is limited to recipients colonized with small conidia Aspergillus species, such as A. fumigatus. ${ }^{19}$

While the colonized patient may be asymptomatic, we hypothesized that Aspergillus colonization causes subclinical injury to the airway epithelium and generates immune responses that promote CLAD pathogenesis. In order to explore the mechanisms responsible, we determined the gene expression profile of bronchoalveolar lavage (BAL) cells during Aspergillus colonization of the lung allograft. Furthermore, we explored the gene expression profile of BAL cells from Aspergillus colonized subjects who develop CLAD as compared to those who remain CLAD free. 


\section{Methods}

\section{Identification of study patients}

Since 2001, a subset of lung transplant recipients at the University of California, Los Angeles (UCLA) was enrolled in an observational registry study that included the collection of BAL fluid for research purposes at the time of standard of care bronchoscopies. This study was approved by the UCLA Institutional Review Board. All subjects provided written informed consent to participate in the study. This study included standardized medical record abstraction including demographic, transplantation, and outcome related variables.

Lung transplant recipients at UCLA undergo surveillance bronchoscopy at 1, 3, 6, and 12 months posttransplant, and when clinically indicated. For this nested case control study, eligible subjects had a 3- or 6-month surveillance bronchoscopy that was negative for AR and negative for infection (BAL culture negative) except for Aspergillus fumigatus, with the corresponding research BAL sample available in our biorepository. Aspergillus colonization was defined as a positive BAL for Aspergillus fumigatus, in the absence of signs or symptoms of infection. CLAD was defined as a sustained drop in $\mathrm{FEV}_{1}$ by at least $20 \%$ from the average of the 2 best posttransplant $\mathrm{FEV}_{1}$ measurements. Incipient CLAD samples were eligible, defined as CLAD within 2 years of sample collection, while delayed CLAD samples were excluded. CLAD free subjects remained without CLAD for at least 3 years following the sample collection.

Our repository included 25 BAL samples from the 3- or 6-month surveillance time-point meeting criteria for Aspergillus fumigatus colonization, of which 5 were excluded for delayed CLAD, and 1 for insufficient data to determine CLAD. Seven additional samples were excluded for degraded ribonucleic acid (RNA), leaving 12 Aspergillus fumigatus colonization cases in this study; 6 progressed to CLAD within 2 years and 6 remained CLAD free for at least 3 years following the sample collection. Our repository included 159 potential samples from the 3- or 6-month surveillance time-point that were culture negative and where the concurrent transbronchial biopsy (TBBX) was negative for AR. We randomly selected 10 representative samples with sufficient RNA quality as controls (16 possible controls screened, 6 excluded for degraded RNA).

\section{Bronchoalveolar lavage fluid sample collection and processing}

The BAL procedure was done according to a standardized protocol using three $60-\mathrm{ml}$ aliquots of isotonic saline instilled into a subsegmental bronchus of either the right middle lobe or left lingula. Retrieved BAL fluid was pooled and then split into a $15 \mathrm{ml}$ clinical specimen and a research specimen with the remaining volume. The freshly acquired research samples were immediately placed on ice for transport to the lab where they were processed within 6 hours of collection. Briefly, the BAL fluid was filtered through sterile gauze, the cells were counted, and cytospin preparations were made for differential cell counts. The remaining cells were separated from fluid by centrifugation. Cells were washed twice with phosphate-buffered saline and lysed in TRIzol (Invitrogen, Carlsbad, CA). 


\section{RNA extraction and microarray analysis}

Total RNA was isolated using TRIzol/chloroform extraction, re-suspended in RNase-free water and purified using the miRNeasy Mini kit (Qiagen Inc, Valencia, CA). RNA was discarded if the 260/280 ratio was not between 1.8 and 2.1, or if RNA showed evidence of degradation (RNA integrity number $<6.5$ ) when assessed with the Agilent 2100 BioAnalyzer (Agilent Technologies, Palo Alto, CA). The poly(A) RNA was converted to cDNA, which was used for in vitro transcription of biotin-labeled cRNA. Hybridization with the biotin labeled RNA, staining, and scanning of the chips followed the procedure outlined in the Affymetrix technical manual.

All analyses used the Affymetrix Human U133 plus 2.0 array, which contains approximately 48,000 probe sets designed from GenBank, dbEST, and RefSeq sequences clustered based on build 133 of the UniGene database and an additional 6500 transcripts identified from Unigene build 159. Background correction utilized the Robust Multi-Array Average method. 20,21 Data were normalized with quantile normalization and Tukey's Median Polish Approach was used to summarize probe intensities. ${ }^{22}$ We focused on probe sets meeting the following criteria: 1) More than 50\% arrays have expression index (log2 scale) of at least 3. This step eliminates probes with low expression index. 2) Coefficient of variation is greater than 0.2 across all arrays. This step excludes probes with low variability. Using these criteria, 28,867 out of 54,675 probes remained after nonspecific filtering.

\section{Statistical methods}

Bioconductor package linear models for microarray data (LIMMA) ${ }^{23}$ was used for differential gene expression analysis (Aspergillus vs no Aspergillus; future CLAD vs No CLAD) adjusted for days to sample. Due to the relatively small number of arrays, empirical Bayesian method is adopted to provide stable testing results. A candidate list of differential expressed probe sets were identified using a volcano plot that showed absolute fold difference $\geq 2.0$ and were significant by LIMMA's moderated t-test $(\mathrm{p}<0.05)$. For functional annotation and pathway enrichment analysis, the candidate probes were analyzed in Database for Annotation, Visualization and Integrated Discovery (DAVID) ${ }^{24,25}$ and processes and pathways were selected based on a FDR less than 5\%. Principal component analysis (PCA) ${ }^{26}$ was used to visualize the separation of the 2 groups based on expression profiles of the selected candidate probes. Further unsupervised hierarchical clustering of differentially expressed probes was done by applying the Ward's minimum variance criterion linkage method ${ }^{27}$ with Euclidean distance and presented in a heat map. PCA and clustering analyses were conducted using genefilter from the Bioconductor suite of packages $^{28}$ in the R statistical software environment version 3.2.3. ${ }^{29}$

\section{Results}

\section{Patient characteristics}

Twenty-two samples from unique subjects were analyzed, including 12 samples with Aspergillus colonization and 10 samples free of infection. Baseline characteristics were similar between groups (Table 1). However, the time from transplant to sample collection 
was significantly longer in the infection free controls (156 \pm 44 days) than the Aspergillus colonized cases ( $103 \pm 36$ days).

\section{BAL cell counts and differential}

We did not make any effort to fractionate cells before RNA isolation. Thus, differences in gene expression could reflect differences in leukocyte cellular proportions within the BAL cell pellets. To assess this, we compared the BAL total cell counts and differential counts between infection free controls and Aspergillus colonized cases. The total cell counts per ml of BAL fluid tended to be higher in Aspergillus colonized cases than infection free controls ( 2.5 vs. $1.3 \times 10^{5}, \mathrm{P}=0.06$ ). Two Aspergillus colonization cases and 1 Aspergillus negative control did not have a cytospin available for differential cell count determination. Among the samples where a cytospin was available, alveolar macrophages were the predominant cell type in every sample. The neutrophil percentages were significantly higher in Aspergillus colonized cases as compared to infection free controls (Figure 1). Lymphocyte percentages also tended to be higher in Aspergillus colonized cases, but this did not meet statistical significance (Figure 1).

\section{Differential Gene Expression Analyses in Aspergillus Colonized vs. Infection Free Groups}

Based upon an absolute fold change $>2.0$ and unadjusted p-value $<0.05$, differential gene expression analysis identified 489 differentially expressed probe sets, which mapped to 404 unique genes, 397 over- and 7 under-expressed in Aspergillus colonization cases as compared to infection free controls (Table S1) (Figure 2).

\section{Functional Annotation and Pathway Enrichment Analyses for Lung Allograft Aspergillus Colonization}

The list of 404 candidate genes was submitted for functional annotation and pathway analysis using DAVID Bioinformatics Resources. Comparison of the biological process category of gene ontology $(\mathrm{GO})$ classification indicated that the predominant processes were related to defense response or response to wounding (Table 2). Similarly, pathways significantly enriched in this gene list were the Kyoto Encyclopedia of Genes and Genomes (KEGG) pathway "cytokine-cytokine receptor interaction" and the Reactome pathway "biological oxidations" (Table 3).

\section{Relationship of gene expression with Aspergillus colonization}

In order to get a visual impression of how the patients were separated based on the selected gene sets, we performed Principle Component Analysis (PCA) using the 404 unique genes differentially expressed. The PCA demonstrated modest separation of Aspergillus colonization from infection free samples (Figure S1A). Likewise, in unsupervised cluster analyses, Aspergillus samples tended to cluster together. Samples segregated into 2 primary clusters: In the first cluster, 7 of 8 samples were Aspergillus colonized. In the second cluster, 9 of 14 samples were infection free (Figure S1B). 


\section{Characteristics of Aspergillus Colonization Cases Who Develop CLAD}

Among Aspergillus colonized cases, 6 progressed to develop CLAD within 2 years of the sample collection date. The remaining 6 were CLAD free for the duration of follow-up and for at least 3 years. Among the CLAD free group, the median time from sample to last follow-up was 1669 (IQR 1520-1961) days. In the Aspergillus colonized cases that developed CLAD, the time from sample to CLAD diagnosis was 633 (IQR 575-675) days. There were no differences in clinical characteristics between groups including the FEV1 percent predicted or FEV1 percent of baseline (Table 4).

The total cell counts per ml of BAL fluid were similar among Aspergillus colonized cases regardless of whether or not they progressed to CLAD (2.7 vs. $\left.2.1 \times 10^{5}, \mathrm{P}=0.29\right)$. Likewise, there were no differences in neutrophil or lymphocyte percentages between cases that progressed to CLAD compared with those who remained CLAD free (Figure 1).

\section{Differential Gene Expression in Aspergillus colonized patients who develop CLAD}

Using the same criteria described above, we next performed differential gene expression analysis between Aspergillus colonization cases who either progressed to CLAD or remained CLAD free for at least 3 years. This analysis identified a list of 69 differentially expressed probe sets, representing 64 unique genes, of which 38 were up-regulated and 26 were down-regulated in patients who progressed to CLAD (Figure 3) (Table S2).

\section{Functional Annotation and Pathway Enrichment Analyses in Aspergillus colonized patients who develop CLAD}

The list of 64 candidate genes was submitted for functional annotation and pathway analysis using DAVID Bioinformatics Resources. ${ }^{25,30}$ Comparison of the biological process category of GO classification indicated that the predominant processes were related to "immune response", with hits also notable for "response to wounding" and "response to cytokine stimulus" (Table 5).

In DAVID pathway analyses, no pathway was significantly enriched in this gene list. However, the gene with the largest fold increase in expression among cases that developed CLAD was small proline-rich protein 3 (SPRR3), which is found in the epidermal differentiation complex and is involved in squamous differentiation. Consistent with this theme, keratin 4 (KRT4) and keratin 13 (KRT13) were also increased in expression in patients who developed CLAD. We also note increased expression of genes related to antifungal innate immunity (hepcidin [HAMP], chitinase-3-like protein 1 [CHI3L1], chitotriosidase [CHIT1]), as well as adaptive immune engagement of T-cells (C-C chemokine receptor type 7 [CCR7], tumor necrosis factor ligand superfamily member 8 [TNFSF8], lymphocyte antigen 9 [LY9]) and B-cells (G Protein-Coupled Receptor 183 [GPR183], Fc $\gamma$ receptor II-b [FCG2RB]) in Aspergillus cases that develop CLAD.

We cross referenced differentially expressed gene lists in both Aspergillus cases relative to controls and in Aspergillus cases that developed CLAD relative to those who remained CLAD free. Eight genes were included in both lists of differentially expressed genes, including SPRR3 and KRT4 (Table 6). 


\section{Cluster analysis of significant genes and relationship}

PCA based on the 64 unique genes differentially expressed demonstrated modest separation of Aspergillus colonized patients who developed CLAD compared to those who remained CLAD free (Figure S2A). In unsupervised cluster analyses, samples clustered into 3 main groups. In the first cluster, all 3 samples developed CLAD. In the second cluster, all 3 samples remained CLAD free. In the third cluster, there were 3 samples that developed CLAD and 3 that remained CLAD free (Figure S2B).

\section{Discussion}

In this study, we describe the transcription profiles in the BAL cell pellet from Aspergillus colonized lung transplant recipients for the first time. Each Aspergillus case included in this study met criteria for asymptomatic "colonization" without overt clinical disease. This is important because we have previously reported that Aspergillus colonization is a risk factor for CLAD. ${ }^{18,19}$ Without direct evidence until now, we have surmised that Aspergillus colonization causes subclinical injury and inflammation, which can initiate and propagate dysregulated repair mechanisms leading to CLAD. In this study, we confirm that Aspergillus colonization is dominated by differential expression of genes pertaining to defense response, response to wounding, and inflammatory response. Furthermore, we show that the expression of genes related to immune response and response to wounding are among those significantly associated with progression to CLAD. Collectively, this study suggests a reparative response to injury may eventually lead to CLAD in some lung transplant recipients with Aspergillus colonization. These findings provide further support of the utility of the BAL cell pellet transcriptome as a tool to examine lung allograft biology.

We know that neutrophils play a key role in the front-line defense against fungal infections.

${ }^{31}$ In immunosuppressed patients, neutrophil functions may be insufficient to eradicate colonization resulting in their persistence in tissues that can cause bystander cell injury. ${ }^{32}$ Neutrophils also amplify inflammatory reactions by release of cytokines and chemokines that activate and recruit other immune cells. ${ }^{32}$ In this study, we confirm that the proportion of neutrophils in the BAL was increased in Aspergillus colonization cases. Furthermore, many of the differentially expressed genes during Aspergillus colonization were related to the recruitment and retention of neutrophils in the allograft. For example, gene expression of the Glu-Leu-Arg (ELR) ${ }^{+}$CXC chemokine neutrophil chemoattractants, CXC chemokine ligand 1 (CXCL1), CXC chemokine ligand 6 (CXCL6), and CXC chemokine ligand 8 (CXCL8), were all increased with Aspergillus colonization, as were CXC chemokine receptor type 1 (CXCR1) and CXC chemokine receptor type 2 (CXCR2), the major CXC chemokine receptors on neutrophils. ${ }^{33}$ Importantly, neutrophil mediated response is critical in limiting invasive infections, ${ }^{34,35}$ and the preservation of this response may explain why most Aspergillus colonization cases postlung transplant do not develop invasive disease.

Given our prior work showing that Aspergillus colonization is a risk factor for CLAD, ${ }^{18,19}$ it is interesting that BAL neutrophilia is also described as a precursor to CLAD. ${ }^{36,37}$ However, in this study, Aspergillus colonized cases who developed CLAD did not have a greater proportion of BAL neutrophils than those who remained CLAD free. Likewise, neutrophil 
mediated processes were not predominant in the list of differentially expressed genes among Aspergillus cases that developed CLAD.

Immune response genes dominate the list associated with the development of CLAD, including markers of innate and adaptive immune responses. Among the markers of innate immunity that are associated with the development of CLAD, CHIT1 and CHI3L1 are of particular interest. Chitin is a component of Aspergillus cell walls, while humans do not biosynthesize chitin. In humans, CHIT1 produced by alveolar macrophages likely aids in the defense against chitin producing pathogens, including Aspergillus. ${ }^{38} \mathrm{CHI} 3 \mathrm{~L} 1$ also binds chitin, but it does not possess chitin hydrolase activity. ${ }^{39} \mathrm{CHI} 3 \mathrm{~L} 1$ is secreted by neutrophils and macrophages and studies have demonstrated that CHI3L1 modulates innate and adaptive immune responses, as well as promotes tissue remodeling. ${ }^{40}$ Collectively, this suggests further exploration of the role for CHIT1 and CHI3L1 in pathogenesis of CLAD is warranted.

GO enrichment analyses also highlight the biologic process of "response to wounding" during Aspergillus colonization in general, as well as in Aspergillus cases that progressed to CLAD. These findings support the potential for persistent subclinical allograft injury occurring during Aspergillus colonization to cause aberrant wound repair and eventually lead to CLAD. Interestingly, we also found epithelial markers in the cell pellets of Aspergillus colonization cases, likely representing injured epithelium disassociated from the basement membrane of allograft airways and alveoli. For example, several differentially expressed genes are suggestive of compensatory squamous differentiation in cases that progress to CLAD. More specifically, SPRR3, which is found in the epidermal differentiation complex and is a marker of squamous metaplasia, was highly expressed in Aspergillus cases that develop CLAD. ${ }^{41}$ Gene expression for keratin 4 and keratin 13, markers of squamous epithelium, were also increased in cases that progress to CLAD. ${ }^{42}$ Moreover, there was augmented expression of HOP homeobox (HOPX), which is restricted to type I alveolar epithelial cells in the lung and has been shown to be up-regulated during wound repair. ${ }^{43}$ These results imply that Aspergillus colonization causing injury to both the airway and alveolar epithelium may eventually lead to CLAD. This is consistent with our prior observation that only small conidia Aspergillus species (eg. A. fumigatus), capable of being deposited in the small airways and alveoli, is a risk factor for CLAD, while large conidia species (eg. A. niger), do not increase the risk of CLAD. ${ }^{19}$

We have recently published on BAL cell pellet gene expression profiles associated with incipient CLAD (ie CLAD within 2 years of BAL sampling). In that study, incipient CLAD was associated with differential expression of genes related to activation and proliferation of cytotoxic lymphocytes (CD8 ${ }^{+} \mathrm{T}$-cells and NK cells). ${ }^{44}$ In the current study, cytotoxic lymphocyte cell pathways were not present in Aspergillus cases that developed CLAD. We suspect that the pathogenesis of CLAD in these cases will evolve, with the expression profiles transitioning from innate immunity to cytotoxic lymphocyte pathways over time. Therefore, a longitudinal study is merited to add further insight into the association between Aspergillus colonization and the development of CLAD. 
There are limitations of this study inherent to the retrospective, single center, cross sectional design. As implied above, it would be valuable to include longitudinal sampling to characterize the evolution of gene expression through the development of CLAD. We demonstrate association between gene expression and either Aspergillus colonization or CLAD, but we cannot delineate the cause-effect direction. Our relatively small sample size also warrants caution in interpreting our findings. We intentionally focused on $A$. fumigatus colonization due to the association with CLAD, and we excluded samples with a positive culture for anything other than A. fumigatus. It is possible that other infections may cause similar gene expression. Finally, our study did not include a validation cohort, and the design of future studies would benefit from inclusion of an external validation cohort. We are somewhat reassured by the fact that the major biologic processes enriched in our gene lists are consistent with expectations.

In summary, we showed that the BAL cell pellet gene expression during Aspergillus colonization is enriched for genes involved in host defense, inflammation, and wound repair. These findings argue that Aspergillus colonization is not entirely benign, and suggest subclinical injury may occur. If unrecognized and untreated, Aspergillus colonization could be responsible for a nonhealing wound of the respiratory epithelium, where reparative mechanisms result in irreversible airway remodeling. In fact, we do find that gene expression indicative epithelial injury is prevalent in cases that progress to CLAD, as are genes related to an immune response. Taken together, the findings imply potential mechanisms explaining our prior observation of increased CLAD risk in lung transplant recipients colonized with Aspergillus. If confirmed in future studies, prevention and/or early treatment of Aspergillus colonization could be a strategy to reduce the risk of CLAD.

\section{Supplementary Material}

Refer to Web version on PubMed Central for supplementary material.

\section{Acknowledgments}

Funding: This study was supported by NIH grants K23HL094746 (PI: SSW), R01HL112990 (PI: JAB), U01AI063594 (PI: SMP) and the UCLA CTSI UL1TR001881.

\section{Abbreviations}

$\begin{array}{ll}\text { AR } & \text { acute rejection } \\ \text { BAL } & \text { bronchoalveolar lavage } \\ \text { BOS } & \text { bronchiolitis obliterans syndrome } \\ \text { CCR7 } & \text { C-C chemokine receptor type 7 } \\ \text { CD30 } & \text { cluster of differentiation marker 30 } \\ \text { CHI3L1 } & \text { chitinase 3-like-1 } \\ \text { CHIT1 } & \text { Chitotriosidase }\end{array}$


CLAD chronic lung allograft dysfunction

CXCL1 CXC chemokine ligand 1

CXCL6 CXC chemokine ligand 6

CXCL8 CXC chemokine ligand 8

CXCR1 CXC chemokine receptor type 1

CXCR2 CXC chemokine receptor type 2

DAVID Database for Annotation, Visualization and Integrated Discovery

ELR Glu-Leu-Arg

FCG2RB Fc $\gamma$ receptor II-b

GO gene ontology

GPR183 G Protein-Coupled Receptor 183

HAMP Hepcidin

HOPX HOP homeobox

IGF-1 insulin like growth factor 1

KEGG Kyoto Encyclopedia of Genes and Genomes

KRT4 keratin 4

KRT13 keratin 13

LIMMA linear models for microarray data

LY9 lymphocyte antigen 9

PCA Principal component analysis

RNA ribonucleic acid

SPRR3 small proline-rich protein 3

TBBX transbronchial biopsy

TNSF8 tumor necrosis factor ligand superfamily member 8

UCLA University of California, Los Angeles

\section{References}

1. Yusen RD, Edwards LB, Dipchand AI, et al. The Registry of the International Society for Heart and Lung Transplantation: Thirty-third Adult Lung and Heart-Lung Transplant Report-2016; Focus Theme: Primary Diagnostic Indications for Transplant. J Heart Lung Transplant. 2016; 35(10): 1170-1184. [PubMed: 27772669] 
2. DerHovanessian A, Todd JL, Zhang A, et al. Validation and Refinement of Chronic Lung Allograft Dysfunction Phenotypes in Bilateral and Single Lung Recipients. Ann Am Thorac Soc. 2016; 13(5): 627-635. [PubMed: 27144793]

3. Sato M, Waddell TK, Wagnetz U, et al. Restrictive allograft syndrome (RAS): a novel form of chronic lung allograft dysfunction. J Heart Lung Transplant. 2011; 30(7):735-742. [PubMed: 21419659]

4. Allyn PR, Duffy EL, Humphries RM, et al. Graft Loss and CLAD-Onset Is Hastened by Viral Pneumonia After Lung Transplantation. Transplantation. 2016; 100(11):2424-2431. [PubMed: 27467538]

5. Botha P, Archer L, Anderson RL, et al. Pseudomonas aeruginosa Colonization of the Allograft After Lung Transplantation and the Risk of Bronchiolitis Obliterans Syndrome. Transplantation. 2008; 85(5):771-774. [PubMed: 18337673]

6. Gregson AL, Wang X, Injean P, et al. Staphylococcus via an interaction with the ELR+CXC chemokine ENA-78 is associated with BOS. Am J Transplant. Mar; 2015 15(3):792-799. [PubMed: 25683785]

7. Gregson AL, Wang X, Weigt SS, et al. Interaction between Pseudomonas and CXC chemokines increases risk of bronchiolitis obliterans syndrome and death in lung transplantation. Am J Respir Crit Care Med. 2013; 187(5):518-526. [PubMed: 23328531]

8. Huang HJ, Yusen RD, Meyers BF, et al. Late primary graft dysfunction after lung transplantation and bronchiolitis obliterans syndrome. Am J Transplant. 2008; 8(11):2454-2462. [PubMed: 18785961]

9. King BJ, Iyer H, Leidi AA, Carby MR. Gastroesophageal reflux in bronchiolitis obliterans syndrome: a new perspective. J Heart Lung Transplant. 2009; 28(9):870-875. [PubMed: 19716037]

10. Snyder LD, Finlen-Copeland CA, Turbyfill WJ, Howell D, Willner DA, Palmer SM. Cytomegalovirus pneumonitis is a risk for bronchiolitis obliterans syndrome in lung transplantation. American journal of respiratory and critical care medicine. 2010; 181(12):13911396. [PubMed: 20167845]

11. Weigt SS, Derhovanessian A, Liao E, et al. CXCR3 chemokine ligands during respiratory viral infections predict lung allograft dysfunction. Am J Transplant. 2012; 12(2):477-484. [PubMed: 22152000]

12. Weigt SS, Elashoff RM, Keane MP, et al. Altered levels of CC chemokines during pulmonary CMV predict BOS and mortality post-lung transplantation. Am J Transplant. 2008; 8(7):15121522. [PubMed: 18513272]

13. Weigt SS, DerHovanessian A, Wallace WD, Lynch JP 3rd, Belperio JA. Bronchiolitis obliterans syndrome: the Achilles' heel of lung transplantation. Semin Respir Crit Care Med. 2013; 34(3): 336-351. [PubMed: 23821508]

14. Bhaskaran A, Hosseini-Moghaddam SM, Rotstein C, Husain S. Mold infections in lung transplant recipients. Seminars in respiratory and critical care medicine. 2013; 34(3):371-379. [PubMed: 23821511]

15. Hsu JL, Khan MA, Sobel RA, et al. Aspergillus fumigatus invasion increases with progressive airway ischemia. PloS one. 2013; 8(10):e77136. [PubMed: 24155924]

16. Nazik H, Penner JC, Ferreira JA, et al. Effects of Iron Chelators on the Formation and Development of Aspergillus fumigatus Biofilm. Antimicrobial agents and chemotherapy. 2015; 59(10):6514-6520. [PubMed: 26239975]

17. Mehrad B, Paciocco G, Martinez FJ, Ojo TC, Iannettoni MD, Lynch JP 3rd. Spectrum of Aspergillus infection in lung transplant recipients: case series and review of the literature. Chest. 2001; 119(1):169-175. [PubMed: 11157600]

18. Weigt SS, Elashoff RM, Huang C, et al. Aspergillus colonization of the lung allograft is a risk factor for bronchiolitis obliterans syndrome. Am J Transplant. 2009; 9(8):1903-1911. [PubMed: 19459819]

19. Weigt SS, Copeland CA, Derhovanessian A, et al. Colonization with small conidia Aspergillus species is associated with bronchiolitis obliterans syndrome: a two-center validation study. Am J Transplant. 2013; 13(4):919-927. [PubMed: 23398785] 
20. Irizarry RA, Bolstad BM, Collin F, Cope LM, Hobbs B, Speed TP. Summaries of Affymetrix GeneChip probe level data. Nucleic Acids Res. 2003; 31(4):e15. [PubMed: 12582260]

21. Irizarry RA, Hobbs B, Collin F, et al. Exploration, normalization, and summaries of high density oligonucleotide array probe level data. Biostatistics. 2003; 4(2):249-264. [PubMed: 12925520]

22. Mosteller, F., Tukey, JW. Data Analysis and Regression A Second Course in Statistics. Reading, MA: Addison-Wesley; 1977.

23. Ritchie ME, Phipson B, Wu D, et al. limma powers differential expression analyses for RNAsequencing and microarray studies. Nucleic Acids Res. 2015; 43(7):e47. [PubMed: 25605792]

24. Ross DJ, Marchevsky A, Kramer M, Kass RM. "Refractoriness" of airflow obstruction associated with isolated lymphocytic bronchiolitis/bronchitis in pulmonary allografts. J Heart Lung Transplant. 1997; 16(8):832-838. [PubMed: 9286775]

25. Huang da W, Sherman BT, Lempicki RA. Systematic and integrative analysis of large gene lists using DAVID bioinformatics resources. Nat Protoc. 2009; 4(1):44-57. [PubMed: 19131956]

26. Jolliffe, IT. Principal Component Analysis. 2. New York: Springer; 2002.

27. Ward JH Jr. Hierarchical Grouping to Optimize an Objective Function. J Am Stat Assoc. 1963; 58(301):236-244.

28. Gentleman RC, Carey VJ, Bates DM, et al. Bioconductor: open software development for computational biology and bioinformatics. Genome Biol. 2004; 5(10):R80. [PubMed: 15461798]

29. Team RC. R: A language and environment for statistical computing. 2015. http://www.Rproject.org/

30. Huang da W, Sherman BT, Lempicki RA. Bioinformatics enrichment tools: paths toward the comprehensive functional analysis of large gene lists. Nucleic Acids Res. 2009; 37(1):1-13. [PubMed: 19033363]

31. Mircescu MM, Lipuma L, van Rooijen N, Pamer EG, Hohl TM. Essential role for neutrophils but not alveolar macrophages at early time points following Aspergillus fumigatus infection. J Infect Dis. 2009; 200(4):647-656. [PubMed: 19591573]

32. Kolaczkowska E, Kubes P. Neutrophil recruitment and function in health and inflammation. Nature reviews Immunology. 2013; 13(3):159-175.

33. Belperio JA, Keane MP, Burdick MD, et al. CXCR2/CXCR2 ligand biology during lung transplant ischemia-reperfusion injury. J Immunol. 2005; 175(10):6931-6939. [PubMed: 16272353]

34. Park SJ, Burdick MD, Mehrad B. Neutrophils mediate maturation and efflux of lung dendritic cells in response to Aspergillus fumigatus germ tubes. Infect Immun. 2012; 80(5):1759-1765. [PubMed: 22392929]

35. Park SJ, Wiekowski MT, Lira SA, Mehrad B. Neutrophils regulate airway responses in a model of fungal allergic airways disease. J Immunol. 2006; 176(4):2538-2545. [PubMed: 16456015]

36. DiGiovine B, Lynch JP 3rd, Martinez FJ, et al. Bronchoalveolar lavage neutrophilia is associated with obliterative bronchiolitis after lung transplantation: role of IL-8. J Immunol. 1996; 157(9): 4194-4202. [PubMed: 8892657]

37. Zheng L, Walters EH, Ward C, et al. Airway neutrophilia in stable and bronchiolitis obliterans syndrome patients following lung transplantation. Thorax. 2000; 55(1):53-59. [PubMed: 10607802]

38. van Eijk M, van Roomen CP, Renkema GH, et al. Characterization of human phagocyte-derived chitotriosidase, a component of innate immunity. Int Immunol. 2005; 17(11):1505-1512. [PubMed: 16214810]

39. Ober C, Chupp GL. The chitinase and chitinase-like proteins: a review of genetic and functional studies in asthma and immune-mediated diseases. Curr Opin Allergy Clin Immunol. 2009; 9(5): 401-408. [PubMed: 19644363]

40. Lee CG, Dela Cruz CS, Herzog E, Rosenberg SM, Ahangari F, Elias JA. YKL-40, a chitinase-like protein at the intersection of inflammation and remodeling. Am J Respir Crit Care Med. 2012; 185(7):692-694. [PubMed: 22467800]

41. Titz B, Sewer A, Schneider T, et al. Alterations in the sputum proteome and transcriptome in smokers and early-stage COPD subjects. J Proteomics. 2015; 128:306-320. [PubMed: 26306861] 
42. van Dorst EB, van Muijen GN, Litvinov SV, Fleuren GJ. The limited difference between keratin patterns of squamous cell carcinomas and adenocarcinomas is explicable by both cell lineage and state of differentiation of tumour cells. J Clin Pathol. 1998; 51(9):679-684. [PubMed: 9930073]

43. Soulet F, Kilarski WW, Antczak P, et al. Gene signatures in wound tissue as evidenced by molecular profiling in the chick embryo model. BMC genomics. 2010; 11:495. [PubMed: 20840761]

44. Weigt SS, Wang X, Palchevskiy V, et al. Gene Expression Profiling of Bronchoalveolar Lavage Cells Preceding a Clinical Diagnosis of Chronic Lung Allograft Dysfunction. PloS one. 2017; 12(1):e0169894. [PubMed: 28103284] 


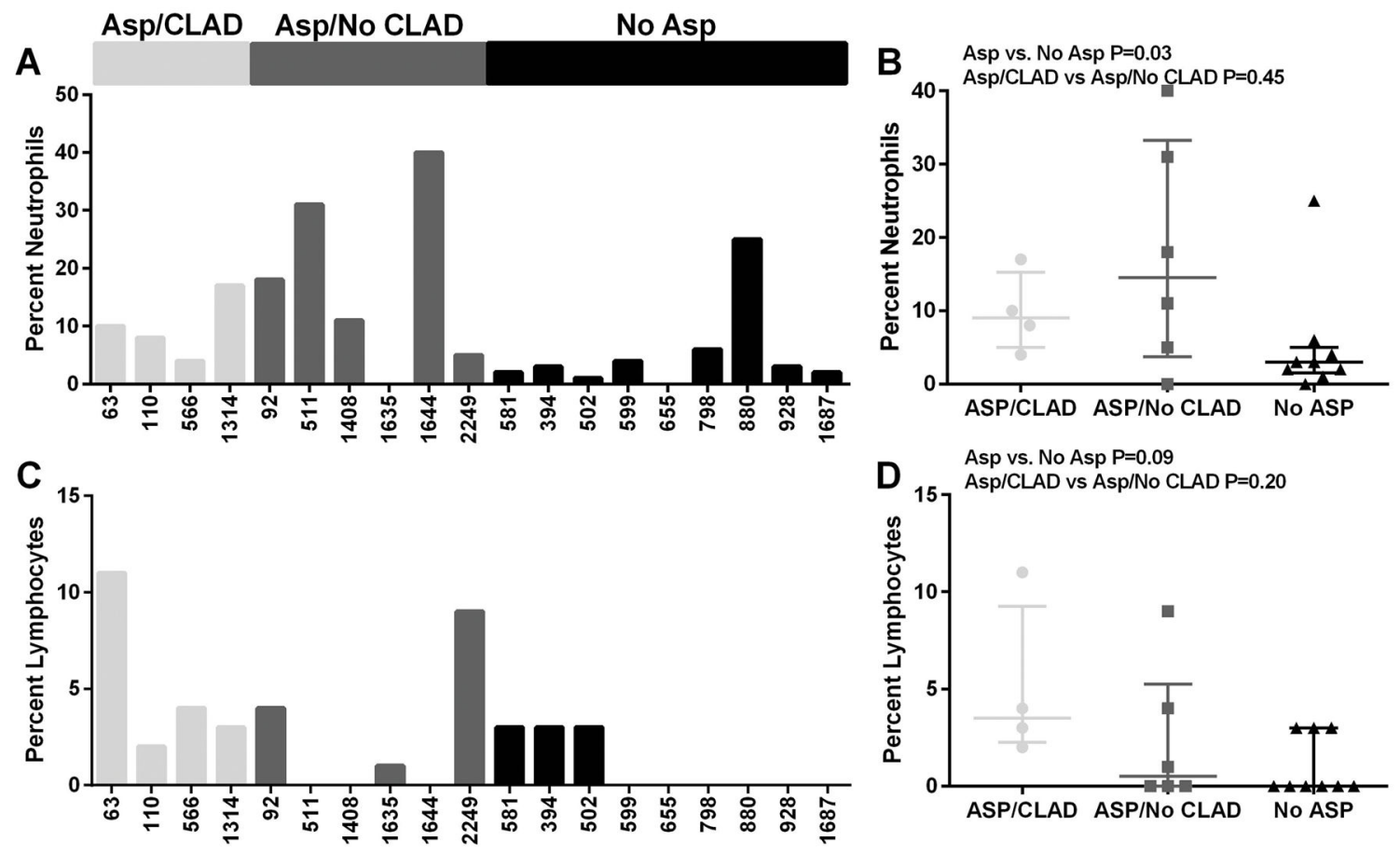

Figure 1. Bronchoalveolar lavage percent leukocyte differentials

(A) Percentage of neutrophils among the BAL cells by sample. (B) Percent neutrophils analysis (Mann-Whitney test) by Aspergillus vs No Aspergillus group. (C) Percent neutrophils analysis (Mann-Whitney test) by Aspergillus/CLAD vs Aspergillus/No CLAD group. (D.) Percentage of lymphocytes among the BAL cells by sample. (E) Percent lymphocytes analysis (Mann-Whitney test) by Aspergillus vs No Aspergillus group. (F) Percent lymphocytes analysis (Mann-Whitney test) by Aspergillus/CLAD vs Aspergillus/No CLAD group. 


\section{Volcano plot}

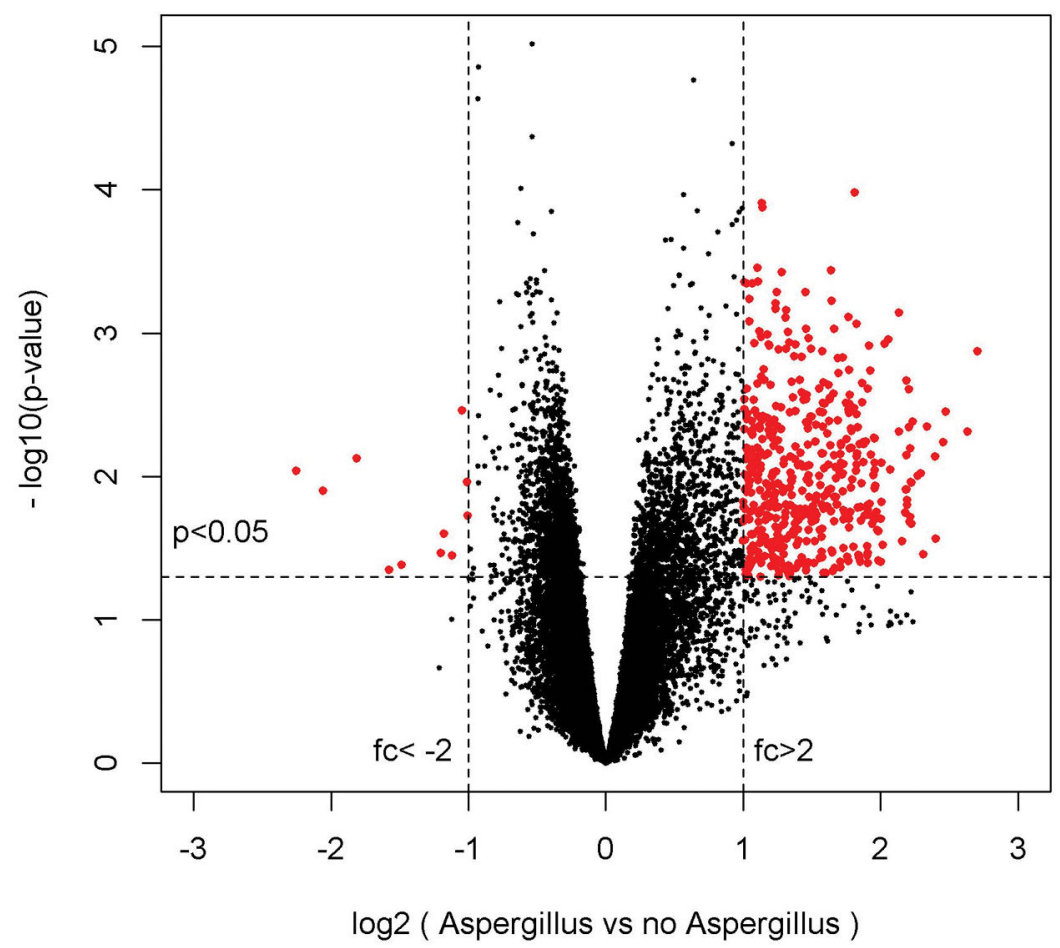

Figure 2.

Volcano Plot visualization of DAE results. DAE generated a candidate list of 489 probe sets in the Aspergillus colonized cases as compared to the infection free controls, based on an unadjusted $\mathrm{P}$-value $<0.05$ and absolute FDR $>2.0$. 
Volcano plot

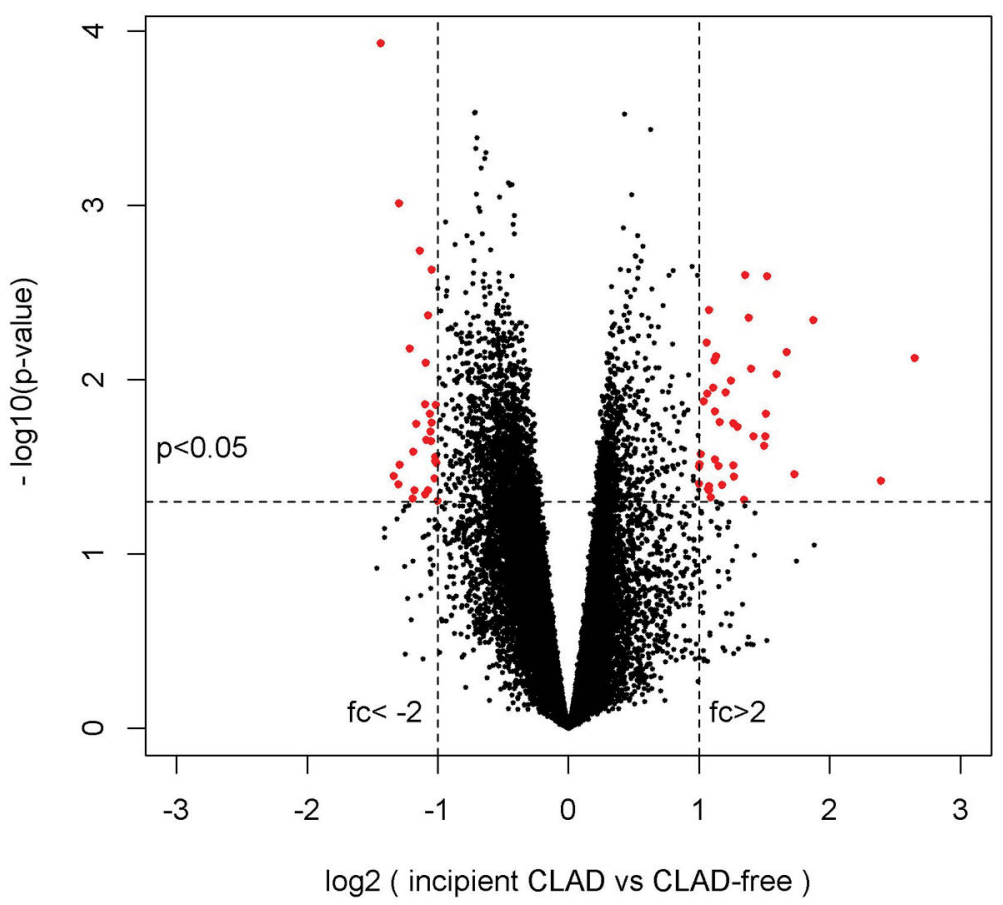

Figure 3.

Volcano Plot visualization of DAE results. DAE generated a candidate list of 69 probe sets in the Aspergillus colonization cases who progressed to CLAD as compared to those who remained CLAD free for at least 3 years, based on an unadjusted P-value $<0.05$ and absolute FDR $>2.0$. 


\section{Table 1}

Clinical characteristics of the No ASP and ASP cohorts.

\begin{tabular}{llll} 
& $\begin{array}{l}\text { No ASP } \\
\text { N=10 }\end{array}$ & $\begin{array}{l}\text { ASP } \\
\text { N=12 }\end{array}$ & P-value \\
\hline Sex & 5 & & 1.0 \\
$\quad$ Male & 5 & 6 & \\
$\quad$ Female & $59(47-61)$ & $62(58-68)$ & 0.11 \\
Age at transplant, median (IQR) & & & 0.78 \\
Pretransplant disease & 2 & 4 & \\
$\quad$ COPD & 7 & 7 & \\
$\quad$ Restrictive lung disease & 1 & 1 & 0.48 \\
$\quad$ Other & & & \\
Type of Transplant & 8 & 8 & 0.65 \\
$\quad$ Bilateral & 2 & 4 & \\
Single & & & \\
Induction Type & 8 & 8 & 0.95 \\
$\quad$ Antithymocyte globulin (ATG) & 2 & 4 & 0.19 \\
$\quad$ Basiliximab & $80(66-100)$ & $79(67-96)$ & \\
FEV1 \% predicted, median (IQR) & $100(97-100)$ & $100(100-100)$ & \\
FEV1 \% of post-transplant baseline, median (IQR) & $156(44)$ & $103(36)$ & 0.01 \\
Days to Sample (SD) & & & \\
\hline
\end{tabular}

Transplantation. Author manuscript; available in PMC 2019 June 01. 
Table 2

Gene ontology: Top biological processes associated with Aspergillus Colonization

\begin{tabular}{lllll}
\hline Term & Count & $\%$ & P Value & FDR $(\%)$ \\
defense response & 36 & 9.1 & $4.38 \mathrm{E}-08$ & 0.00008 \\
response to wounding & 33 & 8.4 & $4.46 \mathrm{E}-08$ & 0.00008 \\
inflammatory response & 25 & 6.3 & $6.16 \mathrm{E}-08$ & 0.0001 \\
regulation of cell proliferation & 39 & 9.9 & $7.59 \mathrm{E}-07$ & 0.001 \\
negative regulation of cell proliferation & 24 & 6.1 & $1.59 \mathrm{E}-06$ & 0.003 \\
cell adhesion & 35 & 8.9 & $2.73 \mathrm{E}-06$ & 0.005 \\
biological adhesion & 35 & 8.9 & $2.78 \mathrm{E}-06$ & 0.005 \\
extracellular space & 35 & 8.9 & $7.82 \mathrm{E}-06$ & 0.01 \\
immune response & 32 & 8.1 & $3.53 \mathrm{E}-05$ & 0.06 \\
plasma membrane & 114 & 28.9 & $8.74 \mathrm{E}-05$ & 0.11 \\
\hline
\end{tabular}

Transplantation. Author manuscript; available in PMC 2019 June 01. 


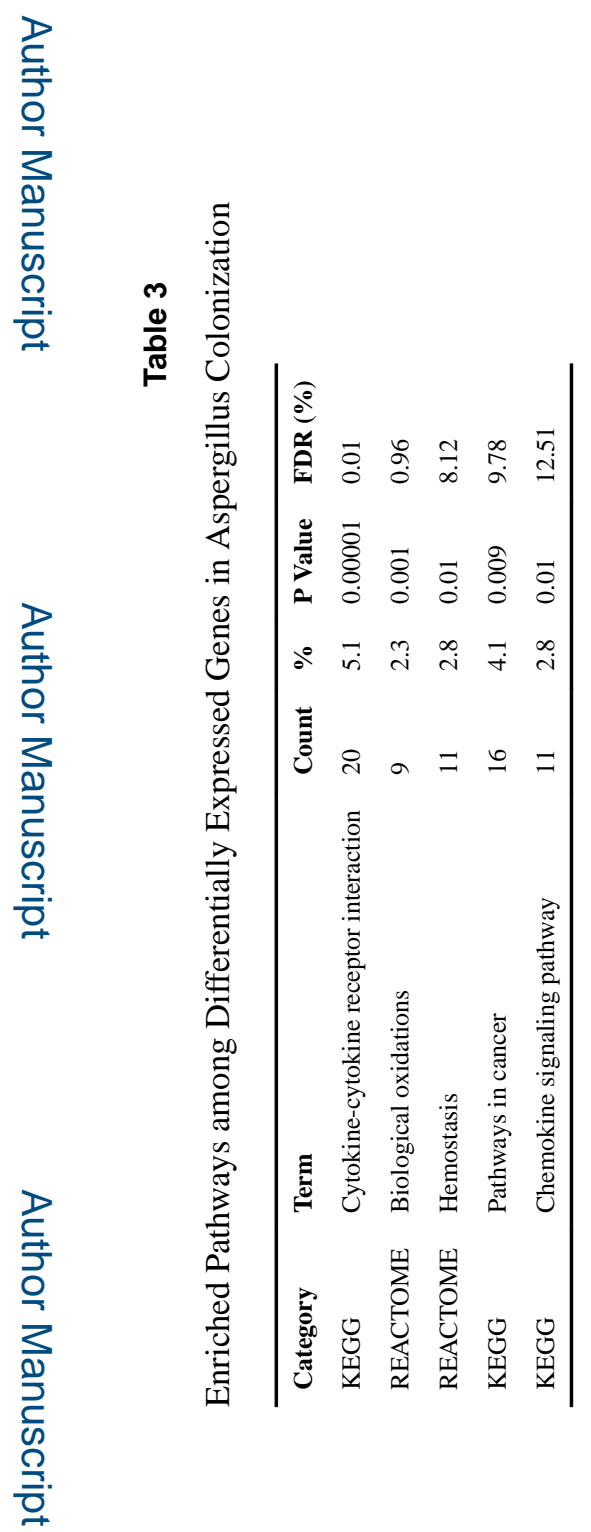

Transplantation. Author manuscript; available in PMC 2019 June 01. 
Table 4

Clinical characteristics of the Aspergillus colonized cases who either developed CLAD or remained CLAD Free.

\begin{tabular}{|c|c|c|c|}
\hline & $\begin{array}{l}\text { CLAD Free } \\
\mathrm{N}=6\end{array}$ & $\begin{array}{l}\text { Developed CLAD } \\
\mathrm{N}=6\end{array}$ & P-value \\
\hline Sex & & & 0.24 \\
\hline Male & 4 & 2 & \\
\hline Female & 2 & 4 & \\
\hline Age at transplant, median (IQR) & $67(54-69)$ & $61(57-63)$ & 0.20 \\
\hline Pretransplant disease & & & 0.28 \\
\hline COPD & 1 & 3 & \\
\hline Restrictive lung disease & 4 & 3 & \\
\hline Other & 1 & 0 & \\
\hline Type of Transplant & & & 0.06 \\
\hline Bilateral & 2 & 6 & \\
\hline Single & 4 & 0 & \\
\hline Induction type & & & 0.55 \\
\hline Antithymocyte globulin (ATG) & 5 & 3 & \\
\hline Basiliximab & 1 & 3 & \\
\hline FEV1 \% predicted, median (IQR) & $74(66-88)$ & 87 (74-97) & 0.63 \\
\hline FEV1 \% of posttransplant baseline, median (IQR) & $100(100-100)$ & $100(100-100)$ & 1.0 \\
\hline Days to Sample (SD) & $122(44)$ & $84(9)$ & 0.09 \\
\hline
\end{tabular}




\section{Table 5}

Gene ontology: Biological processes associated with the development of CLAD in Aspergillus colonized cases.

\begin{tabular}{lllll} 
Term & Count & $\%$ & P Value & FDR $(\%)$ \\
immune response & 12 & 20.0 & 0.00003 & 0.04 \\
protein C-terminus binding & 5 & 8.3 & 0.002 & 1.86 \\
structural molecule activity & 9 & 15.0 & 0.002 & 1.90 \\
regulation of growth & 7 & 11.7 & 0.001 & 2.09 \\
response to wounding & 8 & 13.3 & 0.003 & 4.21 \\
response to cytokine stimulus & 4 & 6.7 & 0.003 & 4.37 \\
wound healing & 5 & 8.3 & 0.005 & 7.36 \\
regulation of locomotion & 5 & 8.3 & 0.005 & 7.49 \\
\hline
\end{tabular}




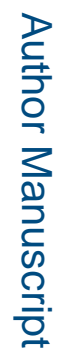

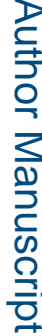

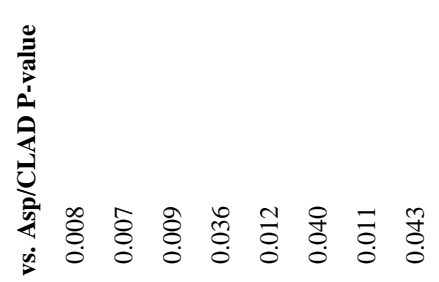

齐

等

范

尝

还

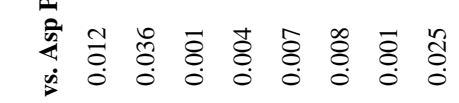
安 z

I

I

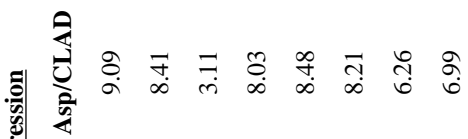
阁

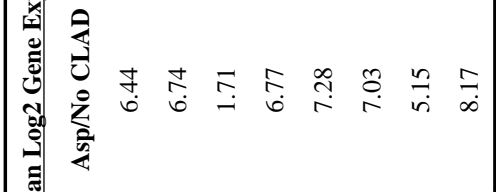

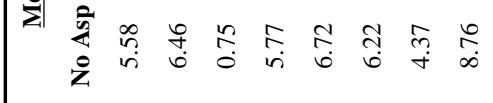

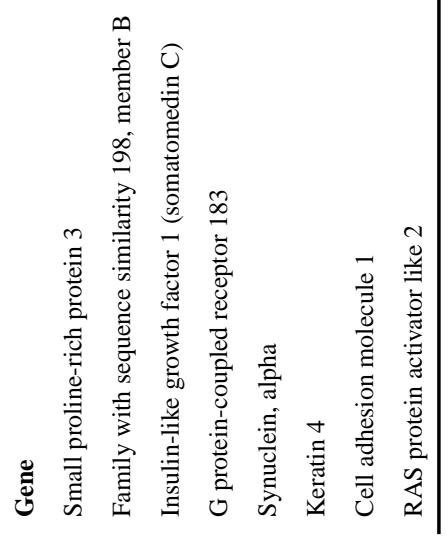

Transplantation. Author manuscript; available in PMC 2019 June 01. 